Louisiana State University

LSU Digital Commons

Faculty Publications

Department of Mathematics

8-9-2018

\title{
Continuous-Discrete Sequential Observers under Sampling and Input Delays
}

Frederic Mazenc

Laboratoire des Signaux et Systèmes

Michael Malisoff

Louisiana State University

Follow this and additional works at: https://digitalcommons.Isu.edu/mathematics_pubs

\section{Recommended Citation}

Mazenc, F., \& Malisoff, M. (2018). Continuous-Discrete Sequential Observers under Sampling and Input Delays. Proceedings of the American Control Conference, 2018-June, 5156-5160. https://doi.org/ 10.23919/ACC.2018.8431518

This Conference Proceeding is brought to you for free and open access by the Department of Mathematics at LSU Digital Commons. It has been accepted for inclusion in Faculty Publications by an authorized administrator of LSU Digital Commons. For more information, please contact ir@lsu.edu. 
archives-ouvertes

\title{
Continuous-Discrete Sequential Observers under Sampling and Input Delays
}

\author{
Frédéric Mazenc, Michael Malisoff
}

\section{To cite this version:}

Frédéric Mazenc, Michael Malisoff. Continuous-Discrete Sequential Observers under Sampling and Input Delays. IEEE American Control Conference (ACC 2018), Jun 2018, Milwaukee, United States. 10.23919/acc.2018.8431518 . hal-01848887

\section{HAL Id: hal-01848887 \\ https://hal.inria.fr/hal-01848887}

Submitted on 25 Jul 2018

HAL is a multi-disciplinary open access archive for the deposit and dissemination of scientific research documents, whether they are published or not. The documents may come from teaching and research institutions in France or abroad, or from public or private research centers.
L'archive ouverte pluridisciplinaire HAL, est destinée au dépôt et à la diffusion de documents scientifiques de niveau recherche, publiés ou non, émanant des établissements d'enseignement et de recherche français ou étrangers, des laboratoires publics ou privés. 


\section{Continuous-Discrete Sequential Observers under Sampling and Input Delays}

\author{
Frederic Mazenc
}

\author{
Michael Malisoff
}

\begin{abstract}
We provide continuous-discrete sequential observers for linear systems with input delays, sampling in the controls and in the outputs, and uncertainties. We also include uncertainties and delays in the output. Our observer allows arbitrarily long constant delays in the input and in the output, and can be used to provide input-to-state stabilizing controllers under uncertain sampled outputs, sampling in the control, and arbitrarily long input and output delays. Since our control does not contain distributed terms, this paper is an extension of the recent prediction technique based on subsequential observers. This provides a useful alternative to existing methods that contained distributed terms. Our example illustrates the large class of systems that are amenable to our methods.
\end{abstract}

Index Terms-Delays, observers, sampling

\section{INTRODUCTION}

The stabilization of linear systems under discrete sampling of an output is a challenging problem with important implications in engineering applications where the output is not available for continuous measurement [8], [26]. This motivated a large literature on delay compensation for systems with outputs and sampling, largely based on prediction or the reduction model approach. The reduction and prediction approaches are notable for their ability to compensate for arbitrarily long input delays, often using dynamic extensions that contain distributed terms; see, e.g., [3], [19], [30].

The work [22] applied the reduction model method to linear systems with piecewise constant inputs, but was confined to cases where there are continuous measurements available for the entire state. Several approaches exist to cope with cases where there are only discrete output measurements. For instance, if the sampling time intervals in the input and the output measurement all have the same length $\bar{c}>0$, and if the input delay is a multiple of $\bar{c}$ and the system is linear and time invariant, then [17] makes it possible to discretize the system so that one can apply a discrete time reduction model approach from [5], [11]. Two other alternative approaches entail (i) combining continuous observers with the reduction model approach and (ii) using continuous-discrete observers. Approach (ii) was used in [18].

Continuous-discrete observers were used in the seminal works [7] and [13], and many more recent works such as [1], [10], [12], [22], [28]. Potential advantages of continuous-discrete observers are that they can be used in

Mazenc is with EPI DISCO INRIA-Saclay, Laboratoire des Signaux et Systèmes (L2S, UMR CNRS 8506), CNRS, CentraleSupélec, Université Paris-Sud, 3 rue Joliot Curie, 91192, Gif-sur-Yvette, France, frederic.mazenc@12s.centralesupelec.fr.

Malisoff is with the Department of Mathematics, Louisiana State University, Baton Rouge, LA 70803-4918, USA, malisoff@1su.edu.

Malisoff was supported by NSF Grants 1408295 and 1711299. cases that are not amenable to continuous observers or static piecewise constant state feedback. The novelty of [18] was that it applied continuous-discrete observers to design globally asymptotically stabilizing output feedback under input delays. This contrasted with other works such as [29] that treated delays, discrete measurements, and sampling separately, with the exception of the nonlinear stabilization results [15], [16] for systems with inputs with zero-order hold and delay and with discrete delayed inputs. For the special case of linear time invariant systems, the feedbacks in [15], [16] are expressed in terms of a sum of past values of the feedback. The work [22] on reduction model methods for linear systems with delayed sampled inputs [9] does not apply to the problem we study in the present work, because [22] requires differentiability of an operator whose analog in the case of impulsive systems would not satisfy the required differentiability conditions.

Here, we build on a series of recent works, which started with [25] and has been developed in [20] and [21]. The fundamental new idea of this approach entails determining future values of the solutions of a system, using a simple dynamic extension where several pointwise delays are present, thus removing the need to introduce distributed delays in the expressions of the control laws or of the dynamic extensions. In the present work, for the first time, this strategy is adapted to the case where continuous-discrete observers are appropriate. Moreover, for the first time, two different delays are taken into account, one in the input and another in the output, under sampling, so we use ideas introduced in [4] to handle systems where the delays are only in the inputs. The proofs of our paper owe a great deal to [18], [22].

The strategy in [18] for stabilization of linear time invariant systems under sampling in both the input and the output was to use a dynamic extension that is similar to the one from [23]. However, works such as [15], [16], [18] can lead to controls with distributed terms that may not always lend themselves to implementations. Therefore, the present work uses a nontrivial variant of our sequential predictor methods from [20], [21] to provide sequential observers and stabilizing feedback controls for linear time invariant systems with sampling in the input, uncertainties in both the output measurements and the original plant, and sampling and uncertainties in the output, which can also ensure inputto-state stability [2] robustness properties under arbitrarily long input and output delays without distributed terms.

In the next section, we state and discuss our main result, and then we prove our result in Section III. Our example in Section IV illustrates the utility of our theory in a detailed 
example, and we conclude in Section $\mathrm{V}$ with an overview of our contribution and our suggestions for future research.

\section{Statement of Main Result}

We study systems of the form

$$
\left\{\begin{aligned}
\dot{x}(t) & =A x(t)+B u(g(t)-\tau)+\delta_{1}(t) \\
y\left(t_{i}\right) & =C x\left(t_{i-r}\right)+\delta_{2}\left(t_{i}\right), \quad i \geq r
\end{aligned}\right.
$$

where $t_{i}=i \nu$ for a known constant $\nu>0$ for all integers $i \geq 0$, the state $x$ and the output $y$ are valued in $\mathbb{R}^{n_{x}}$ and $\mathbb{R}^{n_{y}}$ respectively for any dimensions $n_{x}$ and $n_{y}$, the constants $\tau>0$ and $r \in \mathbb{N}$ and the constant matrices $A, B$, and $C$ are known, $g$ is a right continuous nondecreasing piecewise continuous unbounded function that satisfies $g(t) \leq t$ for all $t$ and so can be used to represent sampling in the control $u$, and the unknown $\delta_{i}$ 's are assumed to be piecewise continuous and bounded and to possess finite right limits at each time $t \geq 0$. We also assume that our initial functions are constant at time $t_{0}=0$. The definition of the sequence $t_{i}$ implies that $y\left(t_{i}\right)=C x\left(t_{i}-r \nu\right)+\delta_{2}\left(t_{i}\right)$. Thus, $y$ has the delay $r \nu$. We assume the following, where $I$ is the identity matrix:

Assumption 1: There is a matrix $L \in \mathbb{R}^{n_{x} \times n_{y}}$ such that

$$
M=(I+L C) e^{\nu A}
$$

is Schur stable, and $\tau / \nu$ is a rational number.

The preceding assumption agrees with [18, Assumption 1] except we added the condition that $\tau / \nu$ is a rational number. Although [18] also allows nonperiodic sampling in the output, [18] uses distributed terms in the controls and so does not cover sequential observers that we present here. By the continuity of the eigenvalues of a matrix as functions of the entries of a matrix, it follows from Assumption 1 that for each sufficiently large integer $d$, the matrix

$$
M_{n}=e^{\nu A}+L C e^{A\left(\nu-\frac{\tau}{d}\right)}
$$

is also Schur stable. We prove the following, where $|\cdot|_{\mathcal{I}}$ denotes the supremum over any interval $\mathcal{I}$, and the superscript + refers to the right limit; see Remark 1 for the definition of solutions for our continuous-discrete systems (4)-(5).

Theorem 1: Let Assumption 1 hold, and choose any positive integers $d>\max \{3, \tau / \nu\}$ and $\ell$ such that $\tau / \nu=d / \ell$ and such that $M_{n}$ in (3) is Schur stable. Set $p=d+\ell r$, and consider the families of continuous-discrete systems

$$
\left\{\begin{array}{l}
\dot{z}_{1}(t)=A z_{1}(t)+B u\left(g\left(t+\frac{\tau}{d}-r \nu\right)-\tau\right) \forall t \in J_{i} \\
z_{1}\left(t_{i}^{+}\right)=z_{1}\left(t_{i}\right)+L\left[C z_{1}\left(t_{i}-\frac{\tau}{d}\right)-y\left(t_{i}\right)\right], i \geq r
\end{array}\right.
$$

and

$$
\left\{\begin{array}{l}
\dot{z}_{j}(t)=A z_{j}(t)+B u\left(g\left(t+\frac{j \tau}{d}-r \nu\right)-\tau\right) \forall t \in J_{i} \\
z_{j}\left(t_{i}^{+}\right)=z_{j}\left(t_{i}\right)+L C \psi_{j}\left(t_{i}-\frac{\tau}{d}\right), i \geq r
\end{array}\right.
$$

for $j=2, \ldots, p$, where $J_{i}=\left(t_{i}, t_{i+1}\right]$ for all $i$ and we set

$$
\begin{aligned}
& \psi_{1}(t)=z_{1}(t)-x\left(t+\frac{\tau}{d}-r \nu\right) \text { and } \\
& \psi_{j}(t)=z_{j}(t)-z_{j-1}\left(t+\frac{\tau}{d}\right) \text { for } j=2, \ldots, p
\end{aligned}
$$

for any choice of the piecewise continuous locally bounded function $u$. Set $\Psi=\left(\psi_{1}, \ldots, \psi_{p}\right)$. Then the following conclusions hold: (a) We can find positive constants $c_{1}$ and $c_{2}$ such that

$$
|\Psi(t)| \leq c_{1}\left(e^{-c_{2} t}\left|\Psi\left(t_{r}^{+}\right)\right|+|\delta|_{[0, t]}\right) \text { for all } t \geq t_{r}
$$

holds along all solutions of (1) and (4)-(5). (b) The equality

$$
z_{p}(t)=x(t+\tau)+\sum_{s=1}^{p} \psi_{s}\left(t+\frac{(p-s) \tau}{d}\right)
$$

holds for all $t \in \mathbb{R}$ along all solutions of (1) and (4)-(5). (c) If, in addition, there is a matrix $K$ such that

$$
\dot{x}(t)=A x(t)+B K x(g(t))+\delta_{a}(t)
$$

is exponentially input-to-state stable with respect to $\delta_{a}$, then the $(x, \Psi)$ dynamics in closed loop with $u(t)=K z_{p}(t)$ are input-to-state stable with respect to $\delta=\left(\delta_{1}, \delta_{2}\right)$.

Remark 1: According to (4)-(5), the $z_{j}$ subsystems are continuous time ones (whose dynamics are defined by the first equations in (4)-(5) for all $t>0$ ), save for the fact that their states are reset to the initial states $z_{j}\left(t_{i}^{+}\right)$at the times $t_{i}$ for all $i \geq r$ when computing $z_{j}(\ell)$ for $\ell$ values on the intervals $J_{i}$. Conclusion (a), in conjunction with conclusion (b), implies that $z_{p}$ provides an observer for the state $x$ of (1), since conclusions (a)-(b) give

$$
\left|z_{p}(t-\tau)-x(t)\right| \leq p c_{1}\left(e^{-c_{2}(t-\tau)}\left|\Psi\left(t_{r}^{+}\right)\right|+\sup _{t \geq 0}|\delta(t)|\right)
$$

for all $t \geq t_{r}+\tau$. Then we can use conclusion (c) to render the $(x, \Psi)$ system input-to-state stable with respect to $\delta$, using our dynamic control that only depends on the perturbed sampled output. When $(A, B)$ is a controllable pair, there are many $g$ 's such that (9) is input-to-state stable with respect to $\delta_{1}$, e.g., if we have sufficiently frequent sampling.

\section{PROOF OF THEOREM 1}

The proof has two parts. First we derive key relations that will enable us to prove the conclusions (a)-(c) of the theorem. In the second part, we use the relations we obtained from the first part of the proof to prove the conclusions (a)-(c).

First Part of Proof. From the definition of the sequence $t_{i}$ and the definition of $y$, we can rewrite (4) as

$$
\begin{gathered}
\dot{z}_{1}(t)=A z_{1}(t)+B u\left(g\left(t+\frac{\tau}{d}-r \nu\right)-\tau\right) \forall t \in J_{i} \\
z_{1}\left(t_{i}^{+}\right)=z_{1}\left(t_{i}\right)+L C\left[z_{1}\left(t_{i}-\frac{\tau}{d}\right)-x\left(t_{i}-r \nu\right)\right] \\
-L \delta_{2}\left(t_{i}\right), i \geq r .
\end{gathered}
$$

Since

$$
\begin{aligned}
& \dot{x}\left(t+\frac{\tau}{d}-r \nu\right)=A x\left(t+\frac{\tau}{d}-r \nu\right) \\
& +B u\left(g\left(t+\frac{\tau}{d}-r \nu\right)-\tau\right)+\delta_{1}\left(t+\frac{\tau}{d}-r \nu\right)
\end{aligned}
$$

holds for all $t \geq-\frac{\tau}{d}+r \nu$, we deduce that $\psi_{1}$ from our definition (6) satisfies

$$
\left\{\begin{aligned}
\dot{\psi}_{1}(t)= & A \psi_{1}(t)-\delta_{1}\left(t+\frac{\tau}{d}-r \nu\right), \forall t \in J_{i} \\
\psi_{1}\left(t_{i}^{+}\right)= & z_{1}\left(t_{i}\right)+L C\left[z_{1}\left(t_{i}-\frac{\tau}{d}\right)-x\left(t_{i}-r \nu\right)\right] \\
& -x\left(t_{i}+\frac{\tau}{d}-r \nu\right)-L \delta_{2}\left(t_{i}\right), i \geq r
\end{aligned}\right.
$$

Hence, if $i \geq r$, then we have

$$
\psi_{1}\left(t_{i}^{+}\right)=\psi_{1}\left(t_{i}\right)+L C \psi_{1}\left(t_{i}-\frac{\tau}{d}\right)-L \delta_{2}\left(t_{i}\right) .
$$


Bearing in mind that $t_{i}-\frac{\tau}{d} \in\left(t_{i-1}, t_{i}\right)$ for all $i$ (which follows from the fact that $t_{i+1}-t_{i}=\nu>\tau / d$ for all $i$ ), we can integrate the first equality in (11) along $\left(t_{i-1}, t_{i}\right]$ and then on $\left(t_{i-1}, t_{i}-\tau / d\right]$, and then substitute the resulting formulas for $\psi_{1}\left(t_{i}\right)$ and $\psi_{1}\left(t_{i}-\tau / d\right)$ into (12), to obtain

$$
\begin{aligned}
\psi_{1}\left(t_{i}^{+}\right)= & e^{A \nu} \psi_{1}\left(t_{i-1}^{+}\right)+L C e^{A\left(\nu-\frac{\tau}{d}\right)} \psi_{1}\left(t_{i-1}^{+}\right) \\
& +\mathcal{N}_{0}(\delta, i) \\
= & M_{n} \psi_{1}\left(t_{i-1}^{+}\right)+\mathcal{N}_{0}(\delta, i),
\end{aligned}
$$

where

$$
\begin{aligned}
\mathcal{N}_{0}(\delta, i)= & -\int_{t_{i-1}}^{t_{i}} \mathcal{M}\left(\delta, t_{i}, \ell\right) \mathrm{d} \ell-L \delta_{2}\left(t_{i}\right) \\
& -L C \int_{t_{i-1}}^{t_{i}-\tau / d} \mathcal{M}\left(\delta, t_{i}-\tau / d, \ell\right) \mathrm{d} \ell
\end{aligned}
$$

and $\mathcal{M}(\delta, s, \ell)=e^{(s-\ell) A} \delta_{1}(\ell+\tau / d-r \nu)$.

Set $z_{0}(t)=x(t-r \nu)$. By our choices (6) of the $\psi_{j}$ 's,

$$
\begin{aligned}
\dot{z}_{j}(t)= & A z_{j}(t) \\
& +B u(\mathcal{G}(t, j)-\tau) \quad \forall t \in J_{i} \\
z_{j}\left(t_{i}^{+}\right)= & z_{j}\left(t_{i}\right) \\
& +L\left[C z_{j}\left(t_{i}-\frac{\tau}{d}\right)-C z_{j-1}\left(t_{i}\right)\right] \\
\dot{z}_{j-1}(t)= & A z_{j-1}(t) \\
& +B u(\mathcal{G}(t, j-1)-\tau) \forall t \in J_{i} \\
z_{j-1}\left(t_{i}^{+}\right)= & z_{j-1}\left(t_{i}\right)-L \Delta_{j-1}\left(t_{i}\right) \\
& +L\left[C z_{j-1}\left(t_{i}-\frac{\tau}{d}\right)-C z_{j-2}\left(t_{i}\right)\right]
\end{aligned}
$$

for all $j \in\{2, \ldots, p\}$ and integers $i \geq r$, where $\mathcal{G}(t, s)=$ $g\left(t+\frac{s \tau}{d}-r \nu\right)$, and where $\Delta_{k}=\delta_{2}$ if $k=1$ and $\Delta_{k}=0$ otherwise. Consequently, since $\tau / d<\nu$,

$$
\left\{\begin{aligned}
\dot{\psi}_{j}(t) & =A \psi_{j}(t) \quad \forall t \in J_{i} \\
\psi_{j}\left(t_{i}^{+}\right) & =\psi_{j}\left(t_{i}\right)+L C \psi_{j}\left(t_{i}-\frac{\tau}{d}\right)
\end{aligned}\right.
$$

holds for all integers $j \in\{2, \ldots, p\}$ and $i \geq r$.

Integrating the first equation in (16) over $\left(t_{i}-\frac{\tau}{d}, t_{i}\right]$ gives

$$
\psi_{j}\left(t_{i}\right)=e^{A \tau / d}\left[z_{j}\left(t_{i}-\frac{\tau}{d}\right)-z_{j-1}\left(t_{i}^{+}\right)\right],
$$

by (6). Bearing (15) in mind, we deduce that

$$
\begin{aligned}
& \psi_{j}\left(t_{i}\right)=e^{A \tau / d}\left[z_{j}\left(t_{i}-\frac{\tau}{d}\right)-z_{j-1}\left(t_{i}\right)\right. \\
& \left.-L C\left[z_{j-1}\left(t_{i}-\frac{\tau}{d}\right)-z_{j-2}\left(t_{i}\right)\right]+L \Delta_{j-1}\left(t_{i}\right)\right]
\end{aligned}
$$

holds for all integers $j \in\{2, \ldots, p\}$ and $i \geq r$. Hence,

$$
\begin{aligned}
\psi_{j}\left(t_{i}\right)= & e^{A \tau / d}\left(\psi_{j}\left(t_{i}-\frac{\tau}{d}\right)+L \Delta_{j-1}\left(t_{i}\right)\right) \\
& -e^{A \tau / d} L C \psi_{j-1}\left(t_{i}-\frac{\tau}{d}\right)
\end{aligned}
$$

holds for all integers $j \in\{2, \ldots, p\}$ and $i \geq r$, so by (16),

$$
\left\{\begin{aligned}
\dot{\psi}_{j}(t)= & A \psi_{j}(t), \quad \forall t \in J_{i} \\
\psi_{j}\left(t_{i}^{+}\right)= & \left(e^{A \tau / d}+L C\right) \psi_{j}\left(t_{i}-\frac{\tau}{d}\right) \\
& -e^{A \tau / d} L C \psi_{j-1}\left(t_{i}-\frac{\tau}{d}\right)+\Delta_{j-1}^{\sharp}\left(t_{i}\right)
\end{aligned}\right.
$$

for all integers $j \in\{2, \ldots, p\}$ and $i \geq r$, where $\Delta_{j-1}^{\sharp}=$ $e^{A \tau / d} L \Delta_{j-1}$. By integrating the first equality of (20) over the interval $\left(t_{i-1}, t_{i}-\tau / d\right]$, it follows that

$$
\left\{\begin{aligned}
\dot{\psi}_{j}(t)= & A \psi_{j}(t), \quad \forall t \in J_{i} \\
\psi_{j}\left(t_{i}^{+}\right)= & {\left[e^{A \tau / d}+L C\right] e^{A\left(\nu-\frac{\tau}{d}\right)} \psi_{j}\left(t_{i-1}^{+}\right) } \\
& -e^{A \tau / d} L C \psi_{j-1}\left(t_{i}-\frac{\tau}{d}\right)+\Delta_{j-1}^{\sharp}\left(t_{i}\right)
\end{aligned}\right.
$$

and therefore also

$$
\left\{\begin{aligned}
\dot{\psi}_{j}(t)= & A \psi_{j}(t), \forall t \in J_{i} \\
\psi_{j}\left(t_{i}^{+}\right)= & M_{n} \psi_{j}\left(t_{i-1}^{+}\right) \\
& -e^{A \tau / d} L C \psi_{j-1}\left(t_{i}-\frac{\tau}{d}\right)+\Delta_{j-1}^{\sharp}\left(t_{i}\right)
\end{aligned}\right.
$$

for all $j \in\{2, \ldots, p\}$ and $i \geq r$, by the definition (3) of $M_{n}$. Since the first lines of (11) and (22) (integrated along the intervals $J_{i}$ ) and the fact that $\tau / d<\nu$ combine to give

$$
\begin{aligned}
& \psi_{1}\left(t_{i}-\frac{\tau}{d}\right)=e^{A(\nu-\tau / d)} \psi_{1}\left(t_{i-1}^{+}\right) \\
& -\int_{t_{i-1}}^{t_{i}-\tau / d} e^{A\left(t_{i}-\tau / d-\ell\right)} \delta_{1}\left(\ell+\frac{\tau}{d}-r \nu\right) \mathrm{d} \ell
\end{aligned}
$$

and

$$
\psi_{j}\left(t_{i}-\frac{\tau}{d}\right)=e^{A(\nu-\tau / d)} \psi_{j}\left(t_{i-1}^{+}\right)
$$

for all integers $i \geq r$ and $j \in\{2, \ldots, p\}$, we can combine (13) with the second equality of (22) to obtain the discrete time system

$$
\left\{\begin{aligned}
\psi_{1}\left(t_{i}^{+}\right)= & M_{n} \psi_{1}\left(t_{i-1}^{+}\right)+\mathcal{N}_{0}(\delta, i) \\
\psi_{j}\left(t_{i}^{+}\right)= & M_{n} \psi_{j}\left(t_{i-1}^{+}\right)+N_{1} \psi_{j-1}\left(t_{i-1}^{+}\right) \\
& +\mathcal{N}_{1}(\delta, j, i)
\end{aligned}\right.
$$

for all integers $i \geq r$ and integers $j \in\{2, \ldots, p\}$, where

$$
N_{1}=-e^{A \tau / d} L C e^{A(\nu-\tau / d)}
$$

and

$$
\begin{aligned}
& \mathcal{N}_{1}(\delta, j, i)=\delta_{2}\left(t_{i}\right) \\
& +e^{A \tau / d} L C \int_{t_{i-1}}^{t_{i}-\tau / d} e^{A\left(t_{i}-\tau / d-\ell\right)} \delta_{1}\left(\ell-\frac{\tau}{d}-r \nu\right) \mathrm{d} \ell
\end{aligned}
$$

when $j=2$ and $\mathcal{N}_{1}(\delta, j, i)=0$ if $3 \leq j \leq p$.

Second Part of Proof. We use the preceding calculations to prove conclusions (a)-(c) of the theorem. Since $M_{n}$ is Schur stable, it follows from the structure of the discrete time system (25) that the dynamics for the discrete time variable

$$
\mathcal{D}(i)=\Psi\left(t_{i}^{+}\right)=\left(\psi_{1}\left(t_{i}^{+}\right), \ldots, \psi_{p}\left(t_{i}^{+}\right)\right)
$$

is exponentially input-to-state stable with respect to $\delta=$ $\left(\delta_{1}, \delta_{2}\right)$, by the following argument. We will use the following discrete time variant of the continuous time recursive Lyapunov function construction from [20], [21] to derive positive constants $c_{a}$ and $c_{b}$ such that

$$
\left|\Psi\left(t_{i}^{+}\right)\right| \leq c_{a}\left(e^{-c_{b}\left(t_{i}-t_{r}\right)}\left|\Psi\left(t_{r}^{+}\right)\right|+|\delta|_{[0, t]}\right)
$$

is satisfied for all integers $i \geq r$. We begin by using standard results (e.g., [27, Lemma 5.7.19]) to find a positive definite matrix $P$ such that

$$
M_{n}^{\top} P M_{n}-P=-I,
$$

and we set

$$
V(x)=x^{\top} P x .
$$

Then substituting in the formula from the right side of the second equation in (25) and then using (30) and Young's inequality and setting

$$
\Delta V(j, i)=V\left(\psi_{j}\left(t_{i}^{+}\right)\right)-V\left(\psi_{j}\left(t_{i-1}^{+}\right)\right)
$$


gives

$$
\begin{aligned}
\Delta V(j, i)=- & \psi_{j}^{\top}\left(t_{i-1}^{+}\right) \psi_{j}\left(t_{i-1}^{+}\right) \\
& +\left(N_{1} \psi_{j-1}\left(t_{i-1}^{+}\right)+\mathcal{N}_{1}(\delta, j, i)\right)^{\top} \\
& \times P\left(N_{1} \psi_{j-1}\left(t_{i-1}^{+}\right)+\mathcal{N}_{1}(\delta, j, i)\right) \\
& +2\left(M_{n} \psi_{j}\left(t_{i-1}^{+}\right)\right)^{\top} \\
& \times P\left(N_{1} \psi_{j-1}\left(t_{i-1}^{+}\right)+\mathcal{N}_{1}(\delta, j, i)\right) \\
\leq & -\left|\psi_{j}\left(t_{i-1}^{+}\right)\right|^{2}+\left|\mathcal{N}_{1}(\delta, j, i)\right|^{2}|P| \\
& +\left|\psi_{j-1}\left(t_{i-1}^{+}\right)\right|^{2}\left|N_{1}\right|^{2}|P| \\
& +\left[2\left\{\left|N_{1}\right||P|\left|\mathcal{N}_{1}(\delta, j, i)\right|\right\}\left\{\left|\psi_{j-1}\left(t_{i-1}^{+}\right)\right|\right\}\right. \\
& +2\left(M_{n} \psi_{j}\left(t_{i-1}^{+}\right)\right)^{\top} \\
& \left.\times P\left(N_{1} \psi_{j-1}\left(t_{i-1}^{+}\right)+\mathcal{N}_{1}(\delta, j, i)\right)\right] \\
\leq & -\frac{1}{2}\left|\psi_{j}\left(t_{i-1}^{+}\right)\right|^{2}+N_{2}\left|\psi_{j-1}\left(t_{i-1}^{+}\right)\right|^{2} \\
& +\mathcal{N}_{2}(\delta, j, i)
\end{aligned}
$$

for $j=2, \ldots, p$ and $i \geq r$, where $N_{2}=\left|N_{1}\right|^{2}|P|+$ $\frac{1}{4}+4\left|M_{n}\right|^{2}|P|^{2}\left|N_{1}\right|^{2}$ and $\mathcal{N}_{2}=\left|\mathcal{N}_{1}\right|^{2}\left(|P|+4\left|N_{1}\right|^{2}|P|^{2}+\right.$ $\left.{ }_{4}\left|M_{n}\right|^{2}|P|^{2}\right)$ and where the second inequality applied the inequality $2 a b \leq \frac{1}{4} a^{2}+4 b^{2}$ three times with suitable $a$ and $b$ (with $a=\left|\psi_{j-1}\left(t_{i-1}^{+}\right)\right|$, and then twice with $a=\left|\psi_{j}\left(t_{i-1}^{+}\right)\right|$) to bound the terms in squared brackets in (33). We also have

$$
\begin{aligned}
\Delta V(1, i)= & -\psi_{1}^{\top}\left(t_{i-1}^{+}\right) \psi_{1}\left(t_{i-1}^{+}\right) \\
& +2\left(M_{n} \psi_{1}\left(t_{i-1}^{+}\right)\right)^{\top} P \mathcal{N}_{0}(\delta, i) \\
& +\mathcal{N}_{0}(\delta, i)^{\top} P \mathcal{N}_{0}(\delta, i) \\
\leq & -\frac{1}{2}\left|\psi_{1}\left(t_{i-1}^{+}\right)\right|^{2}+\mathcal{N}_{3}(\delta, i)
\end{aligned}
$$

where $\mathcal{N}_{3}=\left(2\left|M_{n}\right|^{2}|P|^{2}+|P|\right)\left|\mathcal{N}_{0}\right|^{2}$, and where we used

$$
\begin{aligned}
& 2\left(M_{n} \psi_{1}\left(t_{i-1}^{+}\right)\right)^{\top} P \mathcal{N}_{0}(\delta, i) \leq \\
& \frac{1}{2}\left|\psi_{1}\left(t_{i-1}^{+}\right)\right|^{2}+2\left|M_{n}\right|^{2}|P|^{2}\left|\mathcal{N}_{0}(\delta, i)\right|^{2} .
\end{aligned}
$$

Applying (33) with the choices $j=p$ and then $j=p-1$ and then adding the results gives

$$
\begin{aligned}
& \Delta V(p, i)+\left(2 N_{2}+1\right) \Delta V(p-1, i) \\
& \leq-\frac{1}{2}\left(\left|\psi_{p-1}\left(t_{i-1}^{+}\right)\right|^{2}+\left|\psi_{p}\left(t_{i-1}^{+}\right)\right|^{2}\right) \\
& +2\left(N_{2}+1\right) \overline{\mathcal{N}}_{2}(\delta, i)+\left(2 N_{2}+1\right) N_{2}\left|\psi_{p-2}\left(t_{i-1}^{+}\right)\right|^{2},
\end{aligned}
$$

where $\overline{\mathcal{N}}_{2}(\delta, i)=\max _{j} \mathcal{N}_{2}(\delta, j, i)$. By induction (by multiplying the decay estimate for $\Delta V(p-2, i)$ through by a large enough constant and then using the result to cancel the $\left(2 N_{2}+1\right) N_{2}\left|\psi_{p-2}\left(t_{i-1}^{+}\right)\right|^{2}$ term in (36) and repeating this process), we obtain a nonnegative constant $N_{3}$ and constants $\omega_{i}>0$ for which this exponentially input-to-state stable Lyapunov decay estimate holds along all solutions of the discrete time $\mathcal{D}$ dynamics from (25) for all $i \geq r$ :

$$
\sum_{j=1}^{p} \omega_{i} \Delta V(j, i) \leq-\omega_{0} \sum_{j=1}^{p} \omega_{i} V\left(\psi_{j}\left(t_{i-1}^{+}\right)\right)+N_{3}|\delta|_{[0, t]}^{2}
$$

Then the desired exponentially stable input-to-state stability estimate in the $\mathcal{D}$ variable follows from well known discrete time input-to-state stability results [14]. Conclusion (a) of the theorem follows by combining the exponential input-to-state stability estimate (29) with the fact that the first equalities in (11) and (21) provide a positive constant $\bar{c}$ such that

$$
|\Psi(t)| \leq \bar{c}\left(\left|\Psi\left(t_{i}^{+}\right)\right|+|\delta|_{[0, t]}\right)
$$

for all $t \in J_{i}$ and integers $i \geq 0$, where (38) was used to convert the discrete time decay estimate (29) into a continuous time one in the variable $\Psi(t)$.

To prove the remaining conclusions of the theorem, first note that by our definitions of the $z_{j}$ 's, we deduce that $z_{1}(t)=x\left(t+\frac{\tau}{d}-r \nu\right)+\psi_{1}(t)$ and $z_{j}(t)=z_{j-1}\left(t+\frac{\tau}{d}\right)+$ $\psi_{j}(t)$ for all $j \in\{2, \ldots, p\}$ and $t \geq 0$. It follows by induction on $j \in\{1, \ldots, p\}$ that

$$
z_{p}(t)=x\left(t+\frac{p \tau}{d}-\nu r\right)+\sum_{s=1}^{p} \psi_{s}\left(t+\frac{(p-s) \tau}{d}\right)
$$

for all $t \in \mathbb{R}$. Next recall that our integer $\ell \in \mathbb{N}$ was selected such that $d \nu=\ell \tau$, and that $p-\ell r=d$. This gives

$$
\begin{aligned}
z_{p}(t) & =x\left(t+\frac{p \tau}{d}-\frac{\ell r \tau}{d}\right)+\sum_{s=1}^{p} \psi_{s}\left(t+\frac{(p-s) \tau}{d}\right) \\
& =x(t+\tau)+\sum_{s=1}^{p} \psi_{s}\left(t+\frac{(p-s) \tau}{d}\right),
\end{aligned}
$$

which gives the relation (8) from conclusion (b) of Theorem 1. Conclusion (c) follows from combining conclusions (a)(b) and using the linear structure of the dynamics, by writing the closed loop system from conclusion (c) as

$$
\begin{aligned}
\dot{x}(t) & =A x(t)+B K z_{p}(g(t)-\tau)+\delta_{1}(t) \\
& =A x(t)+B K x(g(t))+\delta_{a}(t)+\delta_{1}(t)
\end{aligned}
$$

for all $t \geq 0$, where

$$
\delta_{a}(t)=B K \sum_{s=1}^{p} \psi_{s}\left(g(t)-\tau+\frac{(p-s) \tau}{d}\right),
$$

and then finding constants $\bar{c}_{i}>0$ and a function $\bar{\gamma} \in \mathcal{K}_{\infty}$ such that $|x(t)| \leq \bar{c}_{1} e^{-\bar{c}_{2} t}|x(t / 2)|+\bar{\gamma}\left(\left|\delta_{a}\right|_{[t / 2, t]}+|\delta|_{[0, t]}\right)$ for all $t \geq 0$ along all solutions of the closed loop system, and finally using the input-to-state stability assumption and conclusion (a) of the theorem and (38) to find a function $\gamma_{*} \in$ $\mathcal{K}_{\infty}$ and a constant $c_{*}>0$ such that $|x(t / 2)| \leq \gamma_{*}(|x(0)|+$ $\left.|\Psi(0)|+|\delta|_{[0, t]}\right)$ and $\left|\delta_{a}\right|_{[t / 2, t]} \leq c_{*}\left(e^{-c_{2} t}|\Psi(0)|+|\delta|_{[0, t]}\right)$ along all solutions of the closed loop system for all $t \geq 0$.

\section{ILLUSTRATION}

Assumption 1 makes Theorem 1 applicable to a broad class of linear time invariant systems, leading to robust continuous-discrete observers that provide stabilizing delay compensating controllers without distributed terms under arbitrarily large constant input and output delays. To illustrate how Theorem 1 can be applied, consider the case where

$$
A=\left[\begin{array}{cc}
0 & 1 \\
-1 & 0
\end{array}\right], C=\left[\begin{array}{ll}
a & b
\end{array}\right], \text { and } \nu=\frac{\pi}{2}+2 k \pi
$$

where $a$ and $b$ are constants and $k$ is any nonnegative integer, for any choice of the matrix $B$. In this case, if we set $L=$

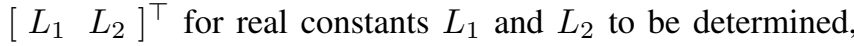
then the matrix $M$ from Assumption 1 takes the form

$$
\begin{aligned}
M & =\left[\begin{array}{cc}
1+L_{1} a & L_{1} b \\
L_{2} a & 1+L_{2} b
\end{array}\right]\left[\begin{array}{cc}
0 & 1 \\
-1 & 0
\end{array}\right] \\
& =\left[\begin{array}{cc}
-L_{1} b & 1+L_{1} a \\
-1-L_{2} b & L_{2} a
\end{array}\right] .
\end{aligned}
$$


Let us find conditions on the $L_{i}$ 's such that (44) is Schur stable, which will make it possible to satisfy Assumption 1 and apply Theorem 1. The matrix (44) has the eigenvalues

$$
\frac{L_{2} a-L_{1} b \pm \sqrt{\left(L_{2} a-L_{1} b\right)^{2}-4\left(1+L_{1} a+L_{2} b\right)}}{2}
$$

which will both be valued in $(-1,1)$ if the conditions

$$
\left\{\begin{array}{l}
L_{1} a+L_{2} b=-1 \\
L_{1} b-L_{2} a \in(-1,1)
\end{array}\right.
$$

are satisfied, and then the continuous-discrete observer is provided by Theorem 1. Viewing (46) as a linear system with unknowns $L_{1}$ and $L_{2}$, we can solve (46) for values $L_{i}$ satisfying (46) if $a$ and $b$ are not both zero. If, in addition, the matrices $B$ and $K$ and the right continuous nondecreasing piecewise continuous unbounded function $g$ are such that $g(t) \leq t$ for all $t \geq 0$ and

$$
\dot{x}(t)=\left[\begin{array}{cc}
0 & 1 \\
-1 & 0
\end{array}\right] x(t)+B K x(g(t))+\delta_{1}(t)
$$

is exponentially input-to-state stable with respect to $\delta_{1}$, then Theorem 1 also provides an input-to-state stabilizing sampled output feedback control.

\section{CONCLUSions}

We developed new observer designs that are motivated by many control engineering applications where only sampled output values are available. We provided a new continuousdiscrete observer design for linear time invariant systems with input delays, uncertainties in the plant, sampling in the output observations, and additive uncertainties on the control and outputs. Unlike [18] and other works that use distributed terms, our sequential observer method produced controllers and stabilizing feedbacks that are free of distributed terms and which therefore may be better suited to implementations. We allow arbitrarily long constant input and output delays, and the input and output delays are allowed to differ. We hope to extend our work to aperiodic sampling in the output, and to use ideas from [15], [24] to cover nonlinear systems. We also hope to use ideas from [6] to cover cases where the matrices $A$ and $B$ in the original system can be uncertain.

\section{REFERENCES}

[1] V. Andrieu and M. Nadri. Observer design for Lipschitz systems with discretetime measurements. In Proceedings of the 49th IEEE Conference on Decision and Control, pages 6522-6527, Atlanta, GA, 2010.

[2] D. Angeli, E. Sontag, and Y. Wang. Input-to-state stability with respect to inputs and their derivatives. International Journal of Robust and Nonlinear Control, 13(11):1035-1056, 2003.

[3] Z. Artstein. Linear systems with delayed controls: A reduction. IEEE Transactions on Automatic Control, 27(4):869-879, 1982.

[4] F. Cacace, A. Germani, and C. Manes. Predictor-based control of linear systems with large and variable measurement delays. International Journal of Control, 87(4):704-714, 2014.

[5] B. Castillo-Toledo, S. Di Gennaro, and G. Sandoval Castro. Stability analysis for a class of sampled nonlinear systems with time-delay. In Proceedings of the 49th IEEE Conference on Decision and Control, pages 1575-1580, Atlanta, GA, 2010.

[6] W-H. Chen, W. Yang, and X. Lu. Impulsive observer-based stabilisation of uncertain linear systems. IET Control Theory and Applications, 3(8):149-159, 2014.
[7] F. Deza, E. Busvelle, J-P. Gauthier, and D. Rakotopara. High gain estimation for nonlinear systems. Systems and Control Letters, 18(4):259-299, 1992.

[8] H. Dinh, R. Kamalapurkar, S. Bhasin, and W. Dixon. Dynamic neural network-based robust observers for uncertain nonlinear systems. Neural Networks, 60:44-52, 2014.

[9] E. Fridman. A refined input delay approach to sampled-data control. Automatica, 46:421-427, 2010.

[10] G. Goffaux, A. Vande Wouwer, and O. Bernard. Improving continuous- discrete interval observers with application to microalgaebased bioprocess. Journal of Process Control, 19(7):1182-1190, 2009.

[11] A. Gonzalez, P. Garcia, P. Albertos, P. Castillo, and R. Lozano. Robustness of a discrete-time predictor-based controller for timevarying measurement delay. Control Engineering Practice, 20(2):102$110,2012$.

[12] H. Hammouri, N. Nadri, and R. Mota. Constant gain observer design for continuous-discrete time uniformly observable systems. In Proceedings of the 45th IEEE Conference on Decision and Control, pages 5406-5411, San Diego, CA, 2006.

[13] A. Jazwinski. Stochastic Processes and Filtering Theory. Dover Publications, Mineola, NY, 1970.

[14] Z-P. Jiang and Y. Wang. Input-to-state stability for discrete-time nonlinear systems. Automatica, 37:857-869, 2001.

[15] I. Karafyllis and M. Krstic. Nonlinear stabilization under sampled and delayed measurements, and with inputs subject to delay and zeroorder hold. IEEE Transcations on Automatic Control, 57(5):1141$1154,2012$.

[16] I. Karafyllis and M. Krstic. Stabilization of nonlinear delay systems using approximate predictors and high-gain observers. Automatica, 49:3623-3631, 2013.

[17] N. Kazantzis, K. Chong, and A. Parlos. Control-relevant discretization of nonlinear systems with time-delay using Taylor-Lie series. ASME Journal of Dynamic Systems, Measurement, and Control, 127(1):153159, 2005.

[18] F. Mazenc and E. Fridman. Predictor-based sampled-data exponential stabilization through continuous-discrete observers. Automatica, 63:74-81, 2016.

[19] F. Mazenc and M. Malisoff. Local stabilization of nonlinear systems through the reduction model approach. IEEE Transactions on Automatic Control, 59(11):3033-3039, 2014.

[20] F. Mazenc and M. Malisoff. Stabilization and robustness analysis for time-varying systems with time-varying delays using a sequential subpredictors approach. Automatica, 82:118-127, 2017.

[21] F. Mazenc and M. Malisoff. Stabilization of nonlinear time-varying systems through a new prediction based approach. IEEE Transcations on Automatic Control, 62(6):2908-2915, 2017.

[22] F. Mazenc and D. Normand-Cyrot. Reduction model approach for linear systems with sampled delayed inputs. IEEE Transcations on Automatic Control, 58(5):1263-1268, 2013.

[23] S. Mondie and W. Michiels. Finite spectrum assignment of unstable timedelay system with a safe implementation. IEEE Transcations on Automatic Control, 48(12):2207-2212, 2003.

[24] M. Nadri, H. Hammouri, and C. Astorga. Observer design for continuous- discrete time state affine systems up to output injection. European Journal of Control, 10(3):252-263, 2004.

[25] M. Najafi, S. Hosseinnia, F. Sheikholeslam, and M. Karimadin. Closed-loop control of dead time systems via sequential subpredictors. International Journal of Control, 86(4):599-609, 2013.

[26] A. Parikh, T.-H. Cheng, H.-Y. Chen, and W. Dixon. A switched systems framework for guaranteed convergence of image-based observers with intermittent measurements. IEEE Transactions on Robotics, 33(2):266-280, 2017

[27] E. Sontag. Mathematical Control Theory. Springer, New York, 1998.

[28] A. Tellez-Anguiano, C. Astorga-Zaragoza, E. Alcorta-Garcia, B. Targui, E. Quintero-Marmol, and M. Adam-Medina et al. Nonlinear continuous-discrete observer application to distillation columns. International Journal of Innovative Computing, Information and Control, 8(1B):763-778, 2012.

[29] H. Wang, Y. Tian, and N. Christov. Piecewise-continuous observers for linear systems with sampled and delayed output. International Journal of Systems Science, 47(8):1804-1815, 2016.

[30] E. Witrant, C. Canudas de Wit, D. Georges, and M. Alamir. Remote stabilization via communication networks with a distributed control law. IEEE Transcations on Automatic Control, 52(8):1480-1485, 2007. 